\title{
Nonlinear stability of Eady's model
}

\author{
Article
}

Published Version

Mu, M. and Shepherd, T. G. (1994) Nonlinear stability of

Eady's model. Journal of the Atmospheric Sciences, 51 (23). pp. 3427-3436. ISSN 1520-0469 doi:

https://doi.org/10.1175/1520-

0469(1994)051<3427:NSOEM>2.0.CO;2 Available at https://centaur.reading.ac.uk/32897/

It is advisable to refer to the publisher's version if you intend to cite from the work. See Guidance on citing.

Published version at: http://dx.doi.org/10.1175/1520-0469(1994)051<3427:NSOEM>2.0.CO;2

To link to this article DOI: http://dx.doi.org/10.1175/1520-

0469(1994)051<3427:NSOEM>2.0.CO;2

Publisher: American Meteorological Society

All outputs in CentAUR are protected by Intellectual Property Rights law, including copyright law. Copyright and IPR is retained by the creators or other copyright holders. Terms and conditions for use of this material are defined in the End User Agreement.

\section{www.reading.ac.uk/centaur}

\section{CentAUR}

Central Archive at the University of Reading

Reading's research outputs online 


\title{
Nonlinear Stability of Eady's Model*
}

\author{
Mu Mu \\ LASG, Institute of Atmospheric Physics, Chinese Academy of Sciences, Beijing, China
}

THEODORE G. SHEPHERD

Department of Physics, University of Toronto, Toronto, Ontario, Canada

(Manuscript received 29 September 1993, in final form 23 March 1994)

\section{ABSTRACT}

A nonlinear stability theorem is established for Eady's model of baroclinic flow. In particular, the Eady basic state is shown to be nonlinearly stable (for arbitrary shear) provided

$$
\frac{\Delta z}{\Delta y}>\frac{2 \sqrt{5} f}{\pi N}
$$

where $\Delta z$ is the height of the domain, $\Delta y$ the channel width, $f$ the Coriolis parameter, and $N$ the buoyancy frequency. When this criterion is satisfied, explicit bounds can be derived on the disturbance potential enstrophy, the disturbance energy, and the disturbance available potential energy on the rigid lids, which are expressed in terms of the initial disturbance fields. The disturbances are completely general (with nonzero potential vorticity) and are not assumed to be of small amplitude. The results may be regarded as an extension of Arnol'd's second nonlinear stability theorem to continuously stratified quasigeostrophic baroclinic flow.

\section{Introduction}

There has been considerable recent interest in the derivation and application of nonlinear stability theorems to quasigeostrophic baroclinic flow. These theorems apply to inviscid, unforced flow and rely on certain exact nonlinear conservation laws, which are associated with the underlying Hamiltonian structure of the dynamics (e.g., Shepherd 1990). For example, by using a suitable combination of momentum and Casimir invariants, a finite-amplitude generalization of the Charney-Stern theorem can be obtained for both layered and continuously stratified flow (Shepherd 1988, 1989). Using a combination of energy and Casimir invariants provides a finite amplitude generalization of the Fjørtoft-Pedlosky theorem (Holm et al. 1985; Swaters 1986; McIntyre and Shepherd 1987; Zeng 1989): this may be regarded as an extension of Arnol'd's $(1965,1966)$ first nonlinear stability theorem to quasigeostrophic flow.

\footnotetext{
* The results of this paper were announced as part of an extended abstract by $\mathrm{Mu}$ and Shepherd (1992).
}

Corresponding author address: Dr. T. G. Shepherd, Department of Physics, University of Toronto, $60 \mathrm{St}$. George Street, Toronto, Ontario M5S 1A7, Canada.
The mathematical method behind the above-mentioned results consists of the construction of a conserved functional, whose first variation at the basic state is zero and whose second variation is sign definite for arbitrary perturbations. The basic state is then an extremum of the conserved functional and is therefore stable. This variational argument was originally introduced by Fjørtoft (1950) and appears to be inherently a small amplitude technique. However, it was realized by Arnol'd (1966), in considering the two-dimensional Euler equations, that in many cases of interest all the higher-order contributions to the functional may be bounded a priori, in which case the stability is in fact nonlinear. It turns out that this insight applies to the quasigeostrophic equations, among others (see above references).

Arnol'd's first stability theorem corresponds to cases where the second variation of the energy-Casimir invariant is positive definite, while the second theorem corresponds to cases where it is negative definite. Establishment of analogues of the first theorem is generally straightforward, but the second theorem turns out to be much more delicate. The difficulty has to do with showing that the positive part of the energy-Casimir invariant is smaller in magnitude than the negative part; this involves the use of a Poincaré inequality, which depends heavily on the presumed boundary conditions. For example, a straightforward extension of Arnol'd's second theorem to continuously stratified flow requires 
that the disturbance have zero circulation on the sidewalls and zero temperature perturbation at the rigid lids [McIntyre and Shepherd (1987), appendix B]: a significant restriction indeed!

The quasigeostrophic analogue of Arnol'd's first theorem, for zonally symmetric flow, requires that $U / Q_{y}$ $<0$ in some frame of reference; here $U$ is the zonal velocity and $Q_{y}$ the meridional gradient of potential vorticity. However, as pointed out by Andrews (1984), most geophysically relevant flows are "pseudoeastward," in the sense that $U / Q_{y}>0$, which takes them into the realm of Arnol'd's second theorem. Of course, one is allowed to choose the frame of reference, and in this way Arnol'd's first theorem may be usefully applied to the Phillips model of baroclinic instability (Shepherd 1993). But with rigid lids, this device is not useful: when interpreting surface temperature gradients as concentrated potential vorticity gradients, in the sense described by the Charney-Stern theorem, it is clear that the Eady basic state cannot be brought into a "pseudowestward" configuration by a change of reference frame. In this case, therefore, one has no choice but to deal with Arnol'd's second theorem directly.

In the case of two-dimensional flow, Mu (1992) and Mu and Shepherd (1994) have recently succeeded in obtaining nonlinear stability criteria analogous to Arnol'd's second theorem. Related results have been obtained for multilayer quasigeostrophic flow by $\mathrm{Mu}$ et al. (1994). The purpose of this paper is to establish a corresponding result for Eady's model of quasigeostrophic baroclinic flow. The work builds on methods developed by Mu and Zeng (1991) and Mu and Wang (1992). When the stability criterion is satisfied, rigorous upper bounds may be derived on the potential enstrophy, the energy, and the boundary available potential energy of finite-amplitude disturbances to the Eady basic state. Unlike the usual normal-mode analysis of the Eady problem, the disturbances considered herein are arbitrary and in particular may have nonzero potential vorticity. The upper bounds hold uniformly in time, are expressed in terms of the initial disturbance fields, and tend to zero uniformly as the initial disturbance amplitude decreases to zero. It follows that the bounds establish nonlinear stability of the Eady basic state.

\section{Statement of the problem}

Consider three-dimensional continuously stratified quasigeostrophic flow on an $f$ plane, governed by the model

$$
\begin{gathered}
\frac{\partial P}{\partial t}+\partial(\Phi, P)=0 \quad \text { in } \Omega \\
\left.\left\{\frac{\partial \Phi_{z}}{\partial t}+\partial\left(\Phi, \Phi_{z}\right)\right\}\right|_{z=z_{i}}=0 \quad \text { in } D \quad[i=0,1]
\end{gathered}
$$

Here $\Phi(x, y, z, t)$ is the streamfunction, and

$$
P=\nabla^{2} \Phi+\frac{1}{S} \Phi_{z z}+f
$$

is the potential vorticity; subscripts denote partial differentiation; $f$ is the (constant) Coriolis parameter; $S=N^{2} / f^{2}$ is a (constant) static stability paranaeter, where $N$ is the buoyancy frequency; $x$ and $y$ are eastward and northward coordinates, $z$ the height coordinate, and $t$ the time; $\partial(a, b) \equiv a_{x} b_{y}-a_{y} b_{x}$ is the two-dimensional Jacobian operator; and $\nabla^{2}=\partial_{x}^{2}+$ $\partial_{y}^{2}$ is the two-dimensional Laplacian operator. The spatial domain is a zonal periodic channel

$$
\begin{aligned}
D=\{-X \leqslant x \leqslant X,-Y \leqslant y \leqslant Y\}, & \\
\Omega & =D \times\left(z_{0}, z_{1}\right),
\end{aligned}
$$

with $0 \leqslant z_{0}<z_{1}<\infty$. The lateral boundary conditions on the channel sidewalls are

$$
\left.\frac{\partial \Phi}{\partial x}\right|_{y=-Y, Y}=0, \frac{d}{d t}\left\{\left.\int_{-X}^{X} \frac{\partial \Phi}{\partial y} d x\right|_{y=-Y, Y}\right\}=0 .(2.4 \mathrm{a}, \mathrm{b})
$$

The system defined by (2.1), (2.2), and (2.4) constitutes the usual Boussinesq $f$-plane quasigeostrophic equations (e.g., Pedlosky 1987). For convenience we take the (constant) reference-state density to be unity. It may be verified that the system has the following integral invariants:

energy

$$
\mathscr{E}[\Phi]=\int_{\Omega} \frac{1}{2}\left\{|\nabla \Phi|^{2}+\frac{1}{S}\left(\Phi_{z}\right)^{2}\right\} d x d y d z
$$

zonal impulse

$$
\mathscr{M}[\Phi]=\int_{\Omega} y P d x d y d z-\left.\frac{1}{S} \int_{D} y \Phi_{z} d x d y\right|_{z=z_{0}} ^{z=z_{1}},
$$

and functionals of the form

$$
\mathscr{C}_{i}[\Phi]=\left.\int_{D} G_{i}\left(\Phi_{z}\right) d x d y\right|_{z=z_{i}}[i=0,1]
$$

(with $G_{i}(\cdot)$ being arbitrary functions), which are Casimir invariants for this problem (e.g., Shepherd 1990 ). Note that $\mathscr{M}[\Phi]$ differs from the zonal momentum $\int_{\Omega} u d x d y d z$ by Casimir invariants; the zonal impulse and zonal momentum are both related to the underlying zonal symmetry in the system (Shepherd 1990).

We now investigate the nonlinear stability of the Eady basic state $\Phi=\Psi=-\Lambda y z, U=-\Psi_{y}=\Lambda z, P$ $=Q=f$, where $\Lambda$ is any given constant. Let $(\psi, q)$ denote a finite-amplitude disturbance to this steady basic state, namely

$\Phi=\Psi+\psi, \quad P=Q+q,\left.\quad \Phi_{z}\right|_{z=z_{i}}=B_{i}+b_{i}$, 
with $\left.B_{i} \equiv \Psi_{z}\right|_{z=z_{i}}=-\Lambda y$. It follows that

$$
q=\nabla^{2} \psi+\frac{1}{S} \psi_{z z}, \quad b_{i}=\left.\psi_{z}\right|_{z=z_{i}} .
$$

In the normal-mode stability problem, one normally takes $q \equiv 0$; however, we do not wish to impose such a restriction. From (2.1a) and the fact that $Q$ is spatially uniform, the disturbance potential vorticity $q$ satisfies

$$
\frac{\partial q}{\partial t}+\partial(\Psi+\psi, q)=0
$$

then with the boundary condition $(2.4 \mathrm{a})$, one obtains

$$
\frac{d}{d t} Z(t) \equiv \frac{d}{d t} \int_{\Omega} \frac{1}{2} q^{2} d x d y d z=0 .
$$

Thus, the disturbance potential enstrophy $Z(t)$ is conserved in time: $Z(t)=Z(0)$. Our aim is now to find a condition under which the disturbance energy,

$$
E(t)=\int_{\Omega} \frac{1}{2}\left\{|\nabla \psi|^{2}+\frac{1}{S}\left(\psi_{z}\right)^{2}\right\} d x d y d z,
$$

and the disturbance available potential energy on the rigid lids,

$$
E_{b}(t)=\int_{D} \frac{1}{2 S}\left\{\left(b_{0}\right)^{2}+\left(b_{1}\right)^{2}\right\} d x d y,
$$

can be bounded in terms of the initial disturbance fields, with the bounds decreasing to zero uniformly as the initial disturbance tends to zero. If this can be done, then the condition is evidently sufficient to establish nonlinear stability of the Eady basic state.

\section{Nonlinear stability}

\section{a. Bounds on disturbance energy}

In this subsection, we use the integral invariants of the dynamics to provide upper bounds on the disturbance energies $E(t)$ and $E_{b}(t)$. First, define the functional

$$
\begin{aligned}
\mathscr{H}[\Phi]=\mathscr{E}[\Phi] & -\alpha \mathscr{M}[\Phi]-\frac{1}{S}\left(\mathscr{C}_{1}[\Phi]-\mathscr{C}_{0}[\Phi]\right) \\
& -\int_{z_{0}}^{z_{1}}\left\{\left.\Psi \int_{-X}^{X} \frac{\partial \Phi}{\partial y} d x\right|_{y=-Y} ^{y=Y}\right\} d z,
\end{aligned}
$$

where $\alpha$ is a constant to be determined later. Since $\mathscr{H}$ consists of a combination of exact invariants together with a term involving the sidewall circulations, which are conserved according to (2.4b), it follows that $\mathscr{H}$ is itself conserved in time. Now define the disturbance functional

$$
H(t)=\mathscr{H}[\Psi+\psi]-\mathscr{H}[\Psi],
$$

which is also an invariant of the motion and which vanishes for zero disturbance. If we take the arbitrary functions $G_{i}(\cdot)$ in the definition of $\mathscr{C}_{i}$ to be given by

$$
G_{0}(\xi)=\frac{\Lambda z_{0}-\alpha}{2 \Lambda} \xi^{2}, \quad G_{1}(\xi)=\frac{\Lambda z_{1}-\alpha}{2 \Lambda} \xi^{2},
$$

then it follows that

$G_{i}\left(B_{i}+b_{i}\right)-G_{i}\left(B_{i}\right)=G_{i}^{\prime}\left(B_{i}\right) b_{i}+\frac{1}{2} G_{i}^{\prime \prime}\left(B_{i}\right)\left(b_{i}\right)^{2}$

with

$$
G_{i}^{\prime}\left(B_{i}\right)=\left.(\Psi+\alpha y)\right|_{z=z_{i}}
$$

and

$$
G_{i}^{\prime \prime}\left(B_{i}\right)=\frac{\Lambda z_{i}-\alpha}{\Lambda} .
$$

Now using (3.2) $-(3.4 a)$, it can be shown (after integrating by parts to eliminate the sidewall circulation terms) that

$$
H(t)=E(t)+A(t)+H_{1}(t),
$$

where $E(t)$ is given by $(2.11)$, and $A(t)$ and $H_{1}(t)$ are defined by

$$
\begin{gathered}
A(t)=-\int_{D} \frac{1}{2 S}\left\{G_{1}^{\prime \prime}\left(B_{1}\right)\left(b_{1}\right)^{2}-G_{0}^{\prime \prime}\left(B_{0}\right)\left(b_{0}\right)^{2}\right\} d x d y \\
H_{1}(t)=-\int_{\Omega}(\Psi+\alpha y) q d x d y d z
\end{gathered}
$$

Now choose $\alpha$ such that

$$
z_{0}<\frac{\alpha}{\Lambda}<z_{1} .
$$

With $\alpha$ satisfying (3.8), it follows that

$$
\begin{gathered}
G_{0}^{\prime \prime}\left(B_{0}\right)=-c_{0} \equiv-\frac{\alpha-\Lambda z_{0}}{\Lambda}<0, \\
G_{1}^{\prime \prime}\left(B_{1}\right)=c_{1} \equiv \frac{\Lambda z_{1}-\alpha}{\Lambda}>0,
\end{gathered}
$$

whence

$$
A(t)=-\int_{D} \frac{1}{2 S}\left\{c_{0}\left(b_{0}\right)^{2}+c_{1}\left(b_{1}\right)^{2}\right\} d x d y,
$$

which is negative definite. Now using the fact that $H(t)$ $=H(0)$, we can write

$-A(t)=E(t)+H_{1}(t)-E(0)-A(0)-H_{1}(0)$.

Equation (3.11) will provide the basis for the upper bounds on disturbance energy, as shown in detail below. 
We note here the parallel, as well as the contrast, with Arnol'd's second stability theorem. The invariant functional being used is $H(t)$, which is not sign definite. Indeed, the first variation of $H(t)$ at the basic state is equal to $H_{1}(t)$, which is generally nonzero no matter how $\alpha$ is chosen. This is quite different from the usual nonlinear-stability methodology, where the invariant functional is specially constructed so that its first variation vanishes at the basic state. However, $H_{1}(t)$ will turn out to be boundable in terms of the initial disturbance [see (3.33)]. This leaves $A(t)$, which is negative definite, and $E(t)$, which is positive definite. The problem then reduces to finding conditions under which $E(t)$ can be bounded by $-A(t)$ plus terms depending only on the initial disturbance. If this can be done, then (3.11) can be rearranged to provide an upper bound for the positive definite quantity $-A(t)$. This is analogous to what is done in proving Arnol'd's second theorem (e.g., McIntyre and Shepherd 1987; Mu and Wang 1992).

To this end, define $\tilde{\psi}=\psi+\varphi$ where

$$
\begin{gathered}
\varphi(z, t)=\frac{\int_{D}\left(\psi_{0}-\psi\right) d x d y}{\int_{D} d x d y}, \\
\psi_{0}(x, y, z)=\psi(x, y, z, 0) .
\end{gathered}
$$

From the definition of $\tilde{\psi}$,

$$
\frac{d}{d t} \int_{D} \tilde{\psi} d x d y=0
$$

Since

$\frac{d}{d t} \int_{D} q d x d y=0$ and

$$
\frac{d}{d t} \int_{D} b_{i} d x d y=0 \quad[i=0,1]
$$

we have that

$$
\varphi_{z z}=0 \quad \text { and }\left.\quad \varphi_{z}\right|_{z=z_{i}}=0 \quad[i=0,1] .
$$

It follows from (3.15) that

$$
\nabla^{2} \tilde{\psi}+\frac{1}{S} \tilde{\psi}_{z z}=q \quad \text { and }\left.\quad \tilde{\psi}_{z}\right|_{z=z_{i}}=b_{i} \quad[i=0,1]
$$

Now multiply ( $3.16 a)$ by $-\tilde{\psi} / 2$ and integrate by parts over $\Omega$, using (3.16b). Noting the inequality

$$
a b \leqslant \frac{\epsilon a^{2}}{2}+\frac{b^{2}}{2 \epsilon}
$$

which holds for any positive constant $\epsilon$, this yields

$$
\begin{aligned}
\int_{\Omega} & \frac{1}{2}\left\{|\nabla \tilde{\psi}|^{2}+\frac{1}{S}\left(\tilde{\psi}_{z}\right)^{2}\right\} d x d y d z \\
\leqslant & \frac{\epsilon}{4} \int_{\Omega} q^{2} d x d y d z+\frac{1}{4 \epsilon} \int_{\Omega} \tilde{\psi}^{2} d x d y d z \\
& +\sum_{i=0}^{1}\left[\frac{\epsilon_{i}}{4} \int_{D} \frac{1}{S}\left(b_{i}\right)^{2} d x d y+\left.\frac{1}{4 \epsilon_{i}} \int_{D} \frac{1}{S} \tilde{\psi}^{2} d x d y\right|_{z=z_{i}}\right] \\
& +\left|H_{*}(t)\right|,
\end{aligned}
$$

where $\epsilon, \epsilon_{0}$, and $\epsilon_{1}$ are positive constants having the appropriate dimensions, to be determined later, and

$$
H_{*}(t)=\frac{1}{2} \int_{z_{0}}^{z_{1}}\left\{\left.\tilde{\psi} \int_{-X}^{X} \frac{\partial \psi_{0}}{\partial y} d x\right|_{y=-Y} ^{y=Y}\right\} d z .
$$

In obtaining the form of $H_{*}(t)$, the lateral boundary conditions (2.4) have been used.

From Mu and Wang [1992, Eqs. (2.19) and (2.20)], we have

$$
\begin{aligned}
\left.\int_{D} \frac{1}{S} \tilde{\psi}^{2} d x d y\right|_{z=z_{i}} \leqslant & F(\chi) \int_{\Omega} \tilde{\psi}^{2} d x d y d z \\
& +\chi \int_{\Omega} \frac{1}{S}\left(\tilde{\psi}_{z}\right)^{2} d x d y d z
\end{aligned}
$$

where

$F(\chi)=F_{1}+\frac{F_{2}}{\chi}, \quad F_{1}=\frac{1}{S\left(z_{1}-z_{0}\right)}, \quad F_{2}=\frac{1}{S}$,

and $\chi$ is an arbitrary positive constant to be determined later. On the other hand, using (3.15) and integrating by parts yields

$$
\int_{\Omega} \frac{1}{S}\left(\tilde{\psi}_{z}\right)^{2} d x d y d z=\int_{\Omega} \frac{1}{S}\left(\psi_{z}\right)^{2} d x d y d z
$$

whence

$$
E(t)=\int_{\Omega} \frac{1}{2}\left\{|\nabla \tilde{\psi}|^{2}+\frac{1}{S}\left(\tilde{\psi}_{z}\right)^{2}\right\} d x d y d z
$$

Using (2.10), (3.20), and (3.22), the inequality (3.18) becomes

$$
\begin{gathered}
E(t) \leqslant \frac{\epsilon}{2} Z(0)+\left[\left(\frac{1}{\epsilon_{0}}+\frac{1}{\epsilon_{1}}\right) \frac{F(\chi)}{4}+\frac{1}{4 \epsilon}\right] \\
\times \int_{\Omega} \tilde{\psi}^{2} d x d y d z+\frac{\chi}{4}\left(\frac{1}{\epsilon_{0}}+\frac{1}{\epsilon_{1}}\right) \int_{\Omega} \frac{1}{S}\left(\tilde{\psi}_{z}\right)^{2} d x d y d z \\
+\sum_{i=0}^{1} \frac{\epsilon_{i}}{4} \int_{D} \frac{1}{S}\left(b_{i}\right)^{2} d x d y+\left|H_{*}(t)\right|
\end{gathered}
$$


To proceed further, we must bound $\int_{\Omega} \tilde{\psi}^{2} d x d y d z$ by using the Poincaré inequality. To this end, let $\lambda_{2}$ be the least positive eigenvalue of the problem

$$
\begin{array}{r}
\nabla^{2} \phi+\lambda \phi=0 \quad \text { in } D,\left.\quad \frac{\partial \phi}{\partial x}\right|_{y=-Y, Y}=0, \\
\left.\int_{-X}^{X} \frac{\partial \phi}{\partial y} d x\right|_{y=-Y, Y}=0 .
\end{array}
$$

$$
\int_{D}\left(\tilde{\psi}-\psi_{0}\right)^{2} d x d y \leqslant \frac{1}{\lambda_{2}} \int_{D}\left|\nabla\left(\tilde{\psi}-\psi_{0}\right)\right|^{2} d x d y
$$

Integrating (3.26) in $z$ then gives

$$
\int_{\Omega} \tilde{\psi}^{2} d x d y d z \leqslant \frac{1}{\lambda_{2}} \int_{\Omega}|\nabla \tilde{\psi}|^{2} d x d y d z+H_{* *}(t)
$$

By the definition of $\tilde{\psi}$, together with (3.13) and (2.4b), we have

$\int_{D}\left(\tilde{\psi}-\psi_{0}\right) d x d y=0,\left.\int_{-X}^{X} \frac{\partial\left(\tilde{\psi}-\psi_{0}\right)}{\partial y} d x\right|_{y=-Y, Y}=0$.

In light of (3.25) and the eigenvalue problem (3.24), the Poincaré inequality yields where

$$
\begin{aligned}
H_{* *}(t) & =\int_{\Omega}\left\{\frac{1}{\lambda_{2}}\left(\left|\nabla \psi_{0}\right|^{2}+2\left|\nabla \psi_{0} \cdot \nabla \tilde{\psi}\right|\right)\right. \\
& \left.+\left(\psi_{0}\right)^{2}+2\left|\psi_{0}\left(\tilde{\psi}-\psi_{0}\right)\right|\right\} d x d y d z
\end{aligned}
$$

Combining (3.23) with (3.27) results in

$$
\begin{aligned}
& {\left[\frac{1}{2}-\left(\frac{1}{\epsilon_{0}}+\frac{1}{\epsilon_{1}}\right) \frac{F(\chi)}{4 \lambda_{2}}-\frac{1}{4 \lambda_{2} \epsilon}\right] \int_{\Omega}|\nabla \tilde{\psi}|^{2} d x d y d z+\left[\frac{1}{2}-\frac{\chi}{4}\left(\frac{1}{\epsilon_{0}}+\frac{1}{\epsilon_{1}}\right)\right] \int_{\Omega} \frac{1}{S}\left(\tilde{\psi}_{z}\right)^{2} d x d y d z} \\
& \leqslant \frac{\epsilon}{2} Z(0)+\sum_{i=0}^{1} \frac{\epsilon_{i}}{4} \int_{D} \frac{1}{S}\left(b_{i}\right)^{2} d x d y+\left|H_{*}(t)\right|+\left[\left(\frac{1}{\epsilon_{0}}+\frac{1}{\epsilon_{1}}\right) \frac{F(\chi)}{4}+\frac{1}{4 \epsilon}\right] H_{* *}(t) .
\end{aligned}
$$

Now let

$$
M=\min \left\{1-\left(\frac{1}{\epsilon_{0}}+\frac{1}{\epsilon_{1}}\right) \frac{F(\chi)}{2 \lambda_{2}}-\frac{1}{2 \lambda_{2} \epsilon}, 1-\frac{\chi}{2}\left(\frac{1}{\epsilon_{0}}+\frac{1}{\epsilon_{1}}\right)\right\},
$$

and assume for now that $M>0$. In this case (3.29) implies

$$
E(t) \leqslant \frac{\epsilon}{2 M} Z(0)+\sum_{i=0}^{1} \frac{\epsilon_{i}}{4 M} \int_{D} \frac{1}{S}\left(b_{i}\right)^{2} d x d y+\frac{1}{M}\left\{\left|H_{*}(t)\right|+\left[\left(\frac{1}{\epsilon_{0}}+\frac{1}{\epsilon_{1}}\right) \frac{F(\chi)}{4}+\frac{1}{4 \epsilon}\right] H_{* *}(t)\right\},
$$

which together with (3.10) and (3.11) yields

$$
\begin{aligned}
\left(c_{0}-\frac{\epsilon_{0}}{2 M}\right) \int_{D} \frac{1}{S}\left(b_{0}\right)^{2} d x d y+\left(c_{1}-\frac{\epsilon_{1}}{2 M}\right) \int_{D} \frac{1}{S}\left(b_{1}\right)^{2} d x d y \leqslant \frac{\epsilon}{M} Z(0)+\frac{2}{M}\left|H_{*}(t)\right| \\
+\frac{2}{M}\left[\left(\frac{1}{\epsilon_{0}}+\frac{1}{\epsilon_{1}}\right) \frac{F(\chi)}{4}+\frac{1}{4 \epsilon}\right] H_{* *}(t)+2\left(E(0)+|A(0)|+\left|H_{1}(0)\right|+\left|H_{1}(t)\right|\right) .
\end{aligned}
$$

This last result provides the bound that we need to prove nonlinear stability. Specifically, if there exist positive constants $\epsilon_{0}, \epsilon_{1}, \epsilon$, and $\chi$ such that $M>0$ and the coefficients of the two integrals on the lefthand side of (3.32) are both positive, then (3.32) provides an upper bound for $E_{b}(t)$. This bound together with (3.31) provides an upper bound for $E(t)$. The question then becomes whether these bounds can be expressed in terms of the initial disturbance. To see this, we need to consider the right-hand side of (3.32). First consider $H_{1}(t)$, which is defined by (3.7): using (2.10) together with the Hölder inequality we have

$$
\begin{aligned}
\left|H_{1}(t)\right| \leqslant\left(\int_{\Omega}(\Psi\right. & \left.+\alpha y)^{2} d x d y d z\right)^{1 / 2} \\
& \times\left(\int_{\Omega}\left(q_{0}\right)^{2} d x d y d z\right)^{1 / 2},
\end{aligned}
$$

where $q_{0}=q(x, y, z, 0)$. In this way $\left|H_{1}(t)\right|$ can be bounded in terms of the initial disturbance field, with the bound tending to zero as the initial disturbance tends to zero. In appendix $\mathrm{A}$, upper bounds on $\left|H_{*}(t)\right|$ and $H_{* *}(t)$ are established in terms of the initial disturbance field, which likewise tend to zero uniformly as the initial disturbance tends to zero. The initial quan- 
tities $E(0),|A(0)|$, and $\left|H_{1}(0)\right|$ all tend to zero in the limit of the initial disturbance tending to zero. Therefore, whenever constants $\epsilon_{0}, \epsilon_{1}, \epsilon$, and $\chi$ exist satisfying the above-mentioned conditions, it follows that the Eady basic state is nonlinearly stable in the sense defined in the introduction.

\section{b. Stability criterion}

The problem now reduces to determining conditions under which there exist constants $\epsilon_{0}, \epsilon_{1}, \epsilon$, and $\chi$ such that

$$
\begin{array}{r}
\epsilon_{0}>0, \quad \epsilon_{1}>0, \quad \epsilon>0, \quad \chi>0, \quad M>0, \\
2 M c_{0}>\epsilon_{0}, \quad \text { and } 2 M c_{1}>\epsilon_{1},
\end{array}
$$

where $M$ is defined by (3.30) and $c_{i}$ are defined by (3.9). It can be shown that a necessary and sufficient condition for (3.34) to hold is that

$$
\lambda_{2}-F_{1}\left(\frac{1}{c_{0}}+\frac{1}{c_{1}}\right)-F_{2}\left(\frac{1}{c_{0}}+\frac{1}{c_{1}}\right)^{2}>0,
$$

where $\lambda_{2}>0$ is defined in terms of the eigenvalue problem (3.24), and $F_{1}$ and $F_{2}$ are positive constants defined by (3.21). That (3.35) implies (3.34) is proved below. The "necessary" part of the statement, that (3.34) implies (3.35), is proved in appendix B.

Thus, suppose that (3.35) holds; if we define $\kappa$ $=\left(1 / c_{0}\right)+\left(1 / c_{1}\right)$, then $(3.35)$ is equivalent to

$$
\lambda_{2}\left(\frac{1}{\kappa}\right)^{2}-F_{1}\left(\frac{1}{\kappa}\right)-F_{2}>0 .
$$

The left-hand side of (3.36) is a quadratic form in $1 /$ $\kappa$, with positive and negative roots; since $1 / \kappa$ is positive, it is clear that the inequality (3.36) can hold only if $1 / \kappa$ is greater than the positive root, namely

$$
\frac{F_{1}+\sqrt{F_{1}^{2}+4 \lambda_{2} F_{2}}}{2 \lambda_{2}}<\frac{1}{\kappa} .
$$

It follows that we may choose $\chi$ in the interval

$$
\frac{F_{1}+\sqrt{F_{1}^{2}+4 \lambda_{2} F_{2}}}{2 \lambda_{2}}<\chi<\frac{1}{\kappa} .
$$

The left-hand inequality of (3.37) implies

$$
\lambda_{2} \chi^{2}-F_{1} \chi-F_{2}>0
$$

this may be equivalently written as $\tau<1$, where $\tau$ $\equiv F(\chi) /\left(\chi \lambda_{2}\right)$, with $F(\chi)$ defined by (3.21). The right-hand inequality of (3.37) implies that $\chi \kappa<1$, which means that we may choose $\delta_{2}$ in the interval

$$
0<\frac{1-\sqrt{1-\chi^{\kappa}}}{2}<\delta_{2}<\frac{1+\sqrt{1-\chi^{\kappa}}}{2}<1 \text {. }
$$

Obviously such a $\delta_{2}$ lies between the two roots of the quadratic $x^{2}-x+\chi \kappa / 4=0$. Therefore,

$$
\delta_{2}^{2}-\delta_{2}+\frac{\chi \kappa}{4}<0
$$

which using the definition of $\kappa$ is equivalent to

$$
\frac{\chi}{4 c_{0}\left(1-\delta_{2}\right)}<\delta_{2}-\frac{\chi}{4 c_{1}\left(1-\delta_{2}\right)} \text {. }
$$

[Note that $\delta_{2}<1$, by (3.39).] In virtue of (3.40), we may choose $\delta_{3}$ such that

$$
\frac{\chi}{4 c_{0}\left(1-\delta_{2}\right)}<\delta_{3}<\delta_{2}-\frac{\chi}{4 c_{1}\left(1-\delta_{2}\right)} \text {. }
$$

Since $0<\tau<1$, we may choose $\delta_{1}$ such that

$$
\tau \delta_{2}<\delta_{1}<\delta_{2} \text {. }
$$

Finally, let

$$
\epsilon=\frac{1}{2 \lambda_{2}\left(\delta_{1}-\tau \delta_{2}\right)}, \quad \epsilon_{0}=\frac{\chi}{2 \delta_{3}}, \quad \epsilon_{1}=\frac{\chi}{2\left(\delta_{2}-\delta_{3}\right)} .
$$

With these choices, it can be seen that $M=\min \{1$ $\left.-\delta_{1}, 1-\delta_{2}\right\}=1-\delta_{2}$. It is not difficult to verify that such $\epsilon, \epsilon_{0}, \epsilon_{1}$, and $\chi$ do indeed satisfy (3.34). Hence (3.35) implies (3.34).

Since the condition (3.35) depends on $\alpha$, the goal is now to choose $\alpha$ in order to get the sharpest possible form of the criterion (3.35), that is, the one holding for the smallest possible value of $\lambda_{2}$. It is clear from the form of (3.35) that this is obtained when $\kappa$ takes its minimum value, which occurs at $\alpha=\Lambda\left(z_{0}+z_{1}\right) / 2$ and is $\kappa=4 /\left(z_{1}-z_{0}\right)$. Note that this value of $\alpha$ lies within the range (3.8), so it is an acceptable choice. Using this value of $\alpha$, and noting that $\lambda_{2}=\pi^{2} / 4 Y^{2},(3.35)$ then implies

$$
\frac{\pi}{2 Y}>\frac{2 \sqrt{5} f}{\left(z_{1}-z_{0}\right) N} .
$$

We may therefore state the following nonlinear stability theorem: If the width of the channel $2 Y$ and the vertical height of the domain $\left(z_{1}-z_{0}\right)$ satisfy (3.44), then the Eady basic state $U=\Lambda z$ is stable to disturbances of arbitrary form and amplitude. In particular, the potential enstrophy, the energy, and the boundary available potential energy of the disturbances are bounded uniformly in time in terms of the initial disturbance, with such bounds decreasing to zero as the initial disturbance decreases to zero. Note that the condition (3.44) is independent of the basic-state shear $\Lambda$.

It is of interest to compare the nonlinear criterion (3.44) with the stability criterion arising in the linear (normal mode) stability theory, which is

$$
\frac{\pi}{2 Y}>\frac{2.40 f}{\left(z_{1}-z_{0}\right) N}
$$

(e.g., Pedlosky 1987). When the condition (3.45) is satisfied, then the Eady basic state is linearly stable to 
disturbances of fixed spatial structure (normal modes) having $q \equiv 0$. Conditions (3.44) and (3.45) are clearly quite similar, differing only by a numerical factor, but their theoretical content is very different. The two criteria are, of course, consistent with one another. Whether the different numerical factor represents something fundamental -in the sense of there being a regime wherein the flow is not subject to normal-mode instability yet is nevertheless unstable in a normed sense - is something the authors do not yet understand. It is a subject that deserves further investigation.

\section{Discussion}

By using exact invariants of the full nonlinear dynamics, a nonlinear stability theorem has been obtained for the Eady basic state $U=\Lambda z$ in the continuously stratified, $f$-plane, Boussinesq model of quasigeostrophic flow. In particular, when the physical parameters of the problem are such that (3.44) is satisfied, then the basic state is nonlinearly stable to disturbances of arbitrary form and magnitude (possibly having nonzero potential vorticity). What is meant by nonlinear stability in this context is that the important quadratic measures of disturbance amplitude - the potential enstrophy, the energy, and the available potential energy on the rigid lids - are all bounded in terms of the initial disturbance, with the bounds decreasing to zero as the initial disturbance decreases to zero. This definition of stability is to be distinguished from the strict mathematical definition of Liapunov stability, which bounds disturbance norms in terms of the initial values of those same norms.

Although the present result may be regarded as an analogue of Arnol'd's second nonlinear stability theorem for this problem, there are some notable differences. In particular, the key inequality (3.32) bounds a quadratic disturbance quantity by a quantity involving a linear disturbance quantity, $H_{1}$; this in turn reflects the fact that the conserved functional $H(t)$ has a nonvanishing first variation at the basic state. Both of these features distinguish the present approach from the energy-Casimir stability methodology of Arnol'd (1965, 1966; see also Holm et al. 1985).

Another difference with Arnol'd's second theorem concerns interpretation. Insofar as the stability criterion (3.44) is a geometric condition, one might be tempted to interpret it as a condition on the scale of the basic flow as compared with the scale of dynamically admissible disturbances. It is well known (McIntyre and Shepherd 1987, appendix B) that in the case of homogeneous boundary conditions, Arnol'd's second theorem can be understood in terms of Fjørtoft's (1953) "anticascade" theorem in precisely this way. However, with inhomogeneous boundary conditions such as arise in Eady's model, the "scale" of the disturbance is not a well-defined concept; thus, an interpretation in terms of the anticascade theorem would seem to be unjustified. This is in contrast to the case of the Phillips model of baroclinic instability, where the analogy with Arnol'd's second theorem is more direct; there the short-wave part of the marginal stability curve coincides precisely with the nonlinear stability threshold (Mu et al. 1994).

The nonlinear stability criterion (3.44) is qualitatively similar to the linear (normal mode) criterion (3.45), differing only by a numerical factor. However, the theoretical content of the two criteria is quite different. Whereas linear stability theorems only delineate parametric regions of possible instability, an important feature of nonlinear stability theorems is that they provide rigorous upper bounds on disturbance norms in terms of the initial conditions of the problem. These bounds may be useful in setting limits on possible nonmodal transient growth, for example. And when the initial flow is unstable but sufficiently close to a stable flow-in a sense that may be made mathematically precise - this feature may be used to determine rigorous upper bounds on the saturation amplitude of the unstable disturbance (Shepherd 1988 et seq.). An application of this method to the present problem could provide an interesting complementary theory to Drazin's (1970) weakly nonlinear analysis of baroclinic instability in the Eady model.

Acknowledgments. This paper was written while MM was visiting the University of Toronto under the auspices of an International Scientific Exchange Award from the Natural Sciences and Engineering Research Council of Canada. MM is supported by the National Natural Science Foundation of China. TGS is supported by NSERC as well as by the Atmospheric Environment Service of Canada.

\section{APPENDIX A}

Upper Bounds on $H_{* *}(t)$ and $\left|H_{*}(t)\right|$

\section{a. The estimate for $H_{* *}(t)$}

From the definition (3.28),

$$
\begin{array}{r}
H_{* *}(t)=\int_{\Omega}\left\{\frac{1}{\lambda_{2}}\left|\nabla \psi_{0}\right|^{2}+\left(\psi_{0}\right)^{2}\right\} d x d y d z \\
+2\left(A_{1}+A_{2}\right)
\end{array}
$$

where

$$
\begin{aligned}
A_{1} & =\int_{\Omega}\left|\psi_{0}\left(\tilde{\psi}-\psi_{0}\right)\right| d x d y d z \\
A_{2} & =\int_{\Omega} \frac{1}{\lambda_{2}}\left|\nabla \psi_{0} \cdot \nabla \tilde{\psi}\right| d x d y d z \\
& =\int_{\Omega} \frac{1}{\lambda_{2}}\left|\nabla \psi_{0} \cdot \nabla \psi\right| d x d y d z
\end{aligned}
$$


Using the Hölder and Poincaré inequalities,

$$
\begin{aligned}
A_{1} \leqslant & \left(\int_{\Omega}\left(\psi_{0}\right)^{2} d x d y d z\right)^{1 / 2}\left(\int_{\Omega}\left(\tilde{\psi}-\psi_{0}\right)^{2} d x d y d z\right)^{1 / 2} \\
\leqslant & \left(\int_{\Omega}\left(\psi_{0}\right)^{2} d x d y d z\right)^{1 / 2} \frac{1}{\sqrt{\lambda_{2}}} \\
& \times\left(\int_{\Omega}\left|\nabla\left(\tilde{\psi}-\psi_{0}\right)\right|^{2} d x d y d z\right)^{1 / 2} \\
= & \frac{1}{\sqrt{\lambda_{2}}}\left(\int_{\Omega}\left(\psi_{0}\right)^{2} d x d y d z\right)^{1 / 2} \\
& \times\left(\int_{\Omega}\left|\nabla(\Psi+\tilde{\psi})-\nabla\left(\Psi+\psi_{0}\right)\right|^{2} d x d y d z\right)^{1 / 2} \\
\leqslant & \frac{\sqrt{2}}{\sqrt{\lambda_{2}}}\left(\int_{\Omega}\left(\psi_{0}\right)^{2} d x d y d z\right)^{1 / 2}\left(\int _ { \Omega } \left\{|\nabla(\Psi+\tilde{\psi})|^{2}\right.\right. \\
& \left.\left.+\left|\nabla\left(\Psi+\psi_{0}\right)\right|^{2}\right\} d x d y d z\right)^{1 / 2} . \quad \text { (A.2) }
\end{aligned}
$$

Now using conservation of total energy (2.5), together with the fact that $\varphi$ does not contribute to the energy, (A.2) implies

$$
\begin{aligned}
A_{1} \leqslant & \frac{\sqrt{2}}{\sqrt{\lambda_{2}}}\left(\int_{\Omega}\left(\psi_{0}\right)^{2} d x d y d z\right)^{1 / 2} \\
& \times\left(\int _ { \Omega } \left\{2\left|\nabla\left(\Psi+\psi_{0}\right)\right|^{2}\right.\right. \\
& \left.\left.\quad+\frac{1}{S}\left[\left(\Psi+\psi_{0}\right)_{z}\right]^{2}\right\} d x d y d z\right)^{1 / 2} .
\end{aligned}
$$

Using similar manipulations,

$$
\begin{aligned}
A_{2} \leqslant & \frac{1}{\lambda_{2}}\left(\int_{\Omega}\left|\nabla \psi_{0}\right|^{2} d x d y d z\right)^{1 / 2} \\
& \times\left(\int_{\Omega}|\nabla \psi|^{2} d x d y d z\right)^{1 / 2} \\
\leqslant & \frac{\sqrt{2}}{\lambda_{2}}\left(\int_{\Omega}\left|\nabla \psi_{0}\right|^{2} d x d y d z\right)^{1 / 2} \\
& \times\left(\int_{\Omega}\left\{|\nabla(\Psi+\psi)|^{2}+|\nabla \Psi|^{2}\right\} d x d y d z\right)^{1 / 2} \\
\leqslant & \frac{\sqrt{2}}{\lambda_{2}}\left(\int_{\Omega}\left|\nabla \psi_{0}\right|^{2} d x d y d z\right)^{1 / 2} \\
& \times\left(\int _ { \Omega } \left\{\left|\nabla\left(\Psi+\psi_{0}\right)\right|^{2}+\frac{1}{S}\left[\left(\Psi+\psi_{0}\right)_{z}\right]^{2}\right.\right. \\
& \left.\left.+|\nabla \Psi|^{2}\right\} d x d y d z\right)^{1 / 2}, \quad \text { (A.4) }
\end{aligned}
$$

again using conservation of total energy. The combination of (A.1), (A.3), and (A.4) demonstrates that
$H_{* *}(t)$ is bounded from above by a quantity depending only on the initial disturbance, which tends to zero as the initial disturbance tends to zero.

\section{b. The estimate for $\left|H_{*}(t)\right|$}

From the definition (3.19) together with the Hölder inequality, we have

$$
\begin{aligned}
\left|H_{*}(t)\right| \leqslant & \frac{1}{2}\left(\left.\int_{z_{0}}^{z_{1}} \tilde{\psi}^{2}\right|_{y=Y} d z\right)^{1 / 2} \\
& \times\left(\int_{z_{0}}^{z_{1}}\left[\left.\int_{-X}^{X} \frac{\partial \psi_{0}}{\partial y} d x\right|_{y=Y}\right]^{2} d z\right)^{1 / 2} \\
& +\frac{1}{2}\left(\left.\int_{z_{0}}^{z_{1}} \tilde{\psi}^{2}\right|_{y=-Y} d z\right)^{1 / 2} \\
& \times\left(\int_{z_{0}}^{z_{1}}\left[\left.\int_{-X}^{X} \frac{\partial \psi_{0}}{\partial y} d x\right|_{y=-Y}\right]^{2} d z\right)^{1 / 2} .
\end{aligned}
$$

The problem now reduces to bounding the integrals of $\tilde{\psi}^{2}$ in the above expression in terms of the initial disturbance. To this end, let $\gamma(y) \equiv(y+Y) /(2 Y)$. First consider $y=Y$ :

$$
\begin{aligned}
{[\tilde{\psi}(x, Y, z, t)]^{2} } & =\int_{-Y}^{Y} \frac{\partial}{\partial y}\left\{\gamma(y) \tilde{\psi}^{2}\right\} d y \\
& =\frac{1}{2 Y} \int_{-Y}^{Y} \tilde{\psi}^{2} d y+\int_{-Y}^{Y} 2 \gamma(y) \tilde{\psi} \tilde{\psi}_{y} d y \\
& \leqslant \frac{2 Y+1}{2 Y} \int_{-Y}^{Y} \tilde{\psi}^{2} d y+\int_{-Y}^{Y}\left(\tilde{\psi}_{y}\right)^{2} d y .
\end{aligned}
$$

The left-hand side of (A.6) is independent of $x$, by the boundary condition ( $2.4 \mathrm{a})$. Noting this, and integrating (A.6) over $x$ and $z$, then yields

$$
\begin{aligned}
\left.\int_{z_{0}}^{z_{1}} \tilde{\psi}^{2}\right|_{y=Y} d z \leqslant \frac{2 Y+1}{4 X Y} \int_{\Omega} \tilde{\psi}^{2} d x d y d z \\
+\frac{1}{2 X} \int_{\Omega}|\nabla \tilde{\psi}|^{2} d x d y d z .
\end{aligned}
$$

Using (3.27) with (A.7), we then have

$$
\begin{aligned}
\left.\int_{z_{0}}^{z_{1}} \tilde{\psi}^{2}\right|_{y=Y} d z \leqslant & \left(\frac{2 Y+1}{4 X Y \lambda_{2}}+\frac{1}{2 X}\right) \\
& \times \int_{\Omega}|\nabla \tilde{\psi}|^{2} d x d y d z+\frac{2 Y+1}{4 X Y} H_{* * *}(t) \\
\leqslant & \left(\frac{2 Y+1}{2 X Y \lambda_{2}}+\frac{1}{X}\right) \int_{\Omega}\left\{\left|\nabla\left(\Psi+\psi_{0}\right)\right|^{2}\right. \\
& \left.+\frac{1}{S}\left[\left(\Psi+\psi_{0}\right)_{z}\right]^{2}+|\nabla \Psi|^{2}\right\} d x d y d z \\
& +\frac{2 Y+1}{4 X Y} H_{* *}(t), \quad \text { (A.8) }
\end{aligned}
$$


the last inequality following from conservation of energy, as in (A.4).

Precisely the same manipulations lead to (A.8) with $y=Y$ on the left-hand side replaced by $y=-Y$. Since $H_{* *}(t)$ has been shown in section a of this appendix to be bounded in terms of the initial disturbance, the combination of (A.5) with (A.8) demonstrates that $\left|H_{*}(t)\right|$ is bounded from above by a quantity depending only on the initial disturbance, which tends to zero as the initial disturbance tends to zero.

\section{APPENDIX B}

\section{Proof That (3.34) Implies (3.35)}

We show below that (3.34) implies (3.35) - equivalently, that the latter condition is necessary for the existence of constants $\epsilon, \epsilon_{0}, \epsilon_{1}$, and $\chi$ satisfying (3.34).

Therefore, suppose that $\epsilon, \epsilon_{0}, \epsilon_{1}$, and $\chi$ exist satisfying (3.34). Let

$$
\begin{aligned}
\delta_{1}=\left(\frac{1}{\epsilon_{0}}+\frac{1}{\epsilon_{1}}\right) \frac{F(\chi)}{2 \lambda_{2}}+\frac{1}{2 \epsilon \lambda_{2}}, \\
\delta_{2}=\frac{\chi}{2}\left(\frac{1}{\epsilon_{0}}+\frac{1}{\epsilon_{1}}\right), \quad \delta_{3}=\frac{\chi}{2 \epsilon_{0}},
\end{aligned}
$$

which is equivalent to (3.43). The hypothesis (3.34) implies

$$
\begin{aligned}
2 M c_{0} & >\frac{\chi}{2 \delta_{3}}, \quad 2 M c_{1}>\frac{\chi}{2\left(\delta_{2}-\delta_{3}\right)}, \\
0 & <\delta_{3}<\delta_{2}<1, \quad \tau \delta_{2}<\delta_{1}<1, \quad \chi>0,
\end{aligned}
$$

where $M=\min \left\{1-\delta_{1}, 1-\delta_{2}\right\}$.

There are two cases to consider. In the case $\delta_{1} \geqslant \delta_{2}$, we have $M=1-\delta_{1}$, and (B.2) thus implies

$$
2 c_{0}\left(1-\delta_{1}\right)>\frac{\chi}{2 \delta_{3}}, \quad 2 c_{1}\left(1-\delta_{1}\right)>\frac{\chi}{2\left(\delta_{2}-\delta_{3}\right)} .
$$

Hence,

$$
\begin{aligned}
\frac{\chi}{4 c_{0}\left(1-\delta_{1}\right)}<\delta_{3}<\delta_{2} & -\frac{\chi}{4 c_{1}\left(1-\delta_{1}\right)} \\
& <\frac{\delta_{1}}{\tau}-\frac{\chi}{4 c_{1}\left(1-\delta_{1}\right)} .
\end{aligned}
$$

The inequality obtained by omitting the two middle terms of (B.3) is equivalent to

$$
\delta_{1}^{2}-\delta_{1}+\frac{\chi \kappa \tau}{4}<0
$$

recalling the definition $\kappa=\left(1 / c_{0}\right)+\left(1 / c_{1}\right)$. Since $\delta_{1}$ $>0$ satisfies the inequality (B.4), the quadratic must have real roots; this implies

$$
1-\chi \kappa \tau>0
$$

which is equivalent to

$$
\lambda_{2}-F_{1} \kappa-\frac{F_{2} \kappa}{\chi}>0
$$

since $\tau=F(\chi) /\left(\chi \lambda_{2}\right)$. On the other hand, (B.3) and the fact that $\delta_{2} \leqslant \delta_{1}$ yields

$$
\frac{\chi}{4 c_{0}\left(1-\delta_{1}\right)}<\delta_{3}<\delta_{1}-\frac{\chi}{4 c_{1}\left(1-\delta_{1}\right)} .
$$

Omitting the middle part of the above inequality produces

$$
\delta_{1}^{2}-\delta_{1}+\frac{\chi \kappa}{4}<0
$$

Since $\delta_{1}>0$ satisfies (B.7), an analogous argument to that concerning (B.4) implies

$$
1-\chi \kappa>0 \Leftrightarrow \kappa<\frac{1}{\chi} .
$$

Combining (B.8) with (B.6) then implies

$$
\lambda_{2}-F_{1} \kappa-F_{2} \kappa^{2}>0,
$$

which is just (3.35).

The second case to consider is $\delta_{1}<\delta_{2}$, for which $M$ $=1-\delta_{2}$ and (B.2) therefore implies

$$
2 c_{0}\left(1-\delta_{2}\right)>\frac{\chi}{2 \delta_{3}}, \quad 2 c_{1}\left(1-\delta_{2}\right)>\frac{\chi}{2\left(\delta_{2}-\delta_{3}\right)} \text {. }
$$

Hence,

$$
\frac{\chi}{4 c_{0}\left(1-\delta_{2}\right)}<\delta_{3}<\delta_{2}-\frac{\chi}{4 c_{1}\left(1-\delta_{2}\right)},
$$

which implies that

$$
\delta_{2}^{2}-\delta_{2}+\frac{\chi \kappa}{4}<0
$$

The fact that $\delta_{2}>0$ satisfies (B.10) then implies (B.8). In this case, since $\tau \delta_{2}<\delta_{1}<\delta_{2}$, it follows that $0<\tau$ $<1$, which is equivalent to

$$
\chi>\frac{F_{1}+\sqrt{F_{1}^{2}+4 \lambda_{2} F_{2}}}{2 \lambda_{2}} .
$$

Combining (B.11) with (B.8) then implies (B.9), which is just (3.35).

\section{REFERENCES}

Andrews, D. G., 1984: On the existence of nonzonal flows satisfying sufficient conditions for stability. Geophys. Astrophys. Fluid Dyn., 28, 243-256.

Arnol'd, V. I., 1965: Conditions for nonlinear stability of stationary plane curvilinear flows of an ideal fluid (in Russian). Dokl. Akad. Nauk. SSSR, 162, 975-978. [English transl.: Sov. Math., 6, 773-777 (1965).]

__ 1966: On an a priori estimate in the theory of hydrodynamical stability (in Russian). Izv. Vyssh. Uchebn. Zaved. Matematika, 
54(5), 3-5. [English transl.: Amer. Math. Soc. Transl., Series 2, 79, 267-269 (1969).]

Drazin, P. G., 1970: Non-linear baroclinic instability of a continuous zonal flow. Quart. J. Roy. Meteor. Soc., 96, 667-676.

Fjørtoft, R., 1950: Application of integral theorems in deriving criteria of stability for laminar flows and for the baroclinic circular vortex. Geofys. Publ., 17(6), 1-52.

,- 1953 : On the changes in the spectral distribution of kinetic energy for two-dimensional, nondivergent flow. Tellus, 5, 225230.

Holm, D. D., J. E. Marsden, T. Ratiu, and A. Weinstein, 1985: Nonlinear stability of fluid and plasma equilibria. Phys. Rep., 123, $1-116$.

McIntyre, M. E., and T. G. Shepherd, 1987: An exact local conservation theorem for finite-amplitude disturbances to non-parallel shear flows, with remarks on Hamiltonian structure and on Arnol'd's stability theorems. J. Fluid Mech., 181, 527-565.

$\mathrm{Mu}$, M., 1992: Nonlinear stability of two-dimensional quasi-geostrophic motions. Geophys. Astrophys. Fluid Dyn., 65, 57-76.

— and Q. Zeng, 1991: Criteria for the nonlinear stability of threedimensional quasi-geostrophic motions. Adv. Atmos. Sci., 8, 110.

—, and T. G. Shepherd, 1992: Nonlinear stability criteria for quasigeostrophic motion. Proc. Fourth Int. Summer Colloquium on Climate, Environment and Geophysical Fluid Dynamics, Beijing, China, Chinese Academy of Sciences, 463-474.
—_ and X. Wang, 1992: Nonlinear stability criteria for the motion of three-dimensional quasigeostrophic flow on a $\beta$-plane. Nonlinearity, 5, 353-371.

—_, and T. G. Shepherd, 1994: On Arnol'd's second nonlinear stability theorem for two-dimensional quasi-geostrophic flow. Geophys. Astrophys. Fluid Dyn., 75, 21-37.

-_, Q. Zeng, T. G. Shepherd, and Y. Liu, 1994: Nonlinear stability of multilayer quasi-geostrophic flow. J. Fluid Mech., 264, 165184.

Pedlosky, J., 1987: Geophysical Fluid Dynamics. 2d ed. SpringerVerlag, $710 \mathrm{pp}$.

Shepherd, T. G., 1988: Nonlinear saturation of baroclinic instability. Part I: The two-layer model. J. Atmos. Sci., 45, 20142025.

___ 1989: Nonlinear saturation of baroclinic instability. Part II: Continuously stratified fluid. J. Atmos. Sci., 46, 888907.

__ 1990: Symmetries, conservation laws, and Hamiltonian structure in geophysical fluid dynamics. Adv. Geophys., 32, 287338.

_- 1993: Nonlinear saturation of baroclinic instability. Part III: Bounds on the energy. J. Atmos. Sci., 50, 2697-2709.

Swaters, G. E., 1986: A nonlinear stability theorem for baroclinic quasi-geostrophic flow. Phys. Fluids, 29, 5-6.

Zeng, Q., 1989: Variational principle of instability of atmospheric motions. Adv. Atmos. Sci., 6, 137-172. 